\title{
Identification of critical genes associated with spinal cord injury based on the gene expression profile of spinal cord tissues from trkB.T1 knockout mice
}

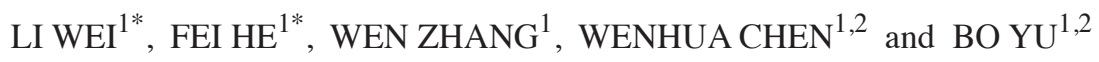 \\ ${ }^{1}$ Department of Rehabilitation, Shanghai General Hospital, Shanghai Jiao Tong University, Shanghai 200080; \\ ${ }^{2}$ School of International Medical Technology, Shanghai Sanda University, Shanghai 201209, P.R. China
}

Received December 28, 2017; Accepted October 3, 2018

DOI: $10.3892 / \mathrm{mmr} .2019 .9884$

\begin{abstract}
The present study aimed to identify the genes and underlying mechanisms critical to the pathology of spinal cord injury (SCI). Gene expression profiles of spinal cord tissues of trkB.T1 knockout (KO) mice following SCI were accessible from the Gene Expression Omnibus database. Compared with trkB.T1 wild type (WT) mice, the differentially expressed genes (DEGs) in trkB.T1 KO mice following injury at different time points were screened out. The significant DEGs were subjected to function, co-expression and protein-protein interaction (PPI) network analyses. A total of 664 DEGs in the sham group and SCI groups at days 1,3 , and 7 following injury were identified. Construction of a Venn diagram revealed the overlap of several DEGs in trkB.T1 KO mice under different conditions. In total, four modules (Magenta, Purple, Brown and Blue) in a co-expression network were found to be significant. Protein tyrosine phosphatase, receptor type C (PTPRC), coagulation factor II, thrombin $(F 2)$, and plasminogen $(P L G)$ were the most significant nodes in the PPI network. ' $\mathrm{Fc} \gamma$ R-mediated phagocytosis' and 'complement and coagulation cascades' were the significant pathways enriched by genes in the PPI and co-expression networks. The results of the present study identified $P T P R C, F 2$ and $P L G$ as potential targets for SCI treatment, which may further improve the general understanding of SCI pathology.
\end{abstract}

Correspondence to: $\mathrm{Dr}$ Bo $\mathrm{Yu}$, Department of Rehabilitation, Shanghai General Hospital, Shanghai Jiao Tong University, 100 Haining Road, Shanghai 200080, P.R. China

E-mail: bobby_JTU@163.com

"Contributed equally

Key words: spinal cord injury, differentially expressed genes, weighted correlation network analysis, protein-protein interaction, significant nodes

\section{Introduction}

Spinal cord injury (SCI) is a disabling condition with significant morbidity and mortality, that affects $\sim 11,000$ individuals annually worldwide (1). The mean age for patients with SCI is 33 years worldwide, and the incidence of SCI is approximately four times higher among males (2). Spinal cord damage may result in pain and paralysis, as well as loss of sensation and physical function. The causes of SCI are varied and include accidents, falls, infections and tumors (3). In addition, patients with SCI may develop spondylosis, a common degenerative change in the cervical spine (4) and its incidence has increased over the past several decades, but management strategies to reduce SCI prevalence have not yet been developed.

The current management strategies for SCI are limited. A better understanding of the physiological mechanisms underlying the disease may lead to the identification of novel interventions. A study exploring the mechanisms underlying SCI has reported that overexpression of signal transducer and activator of transcription 3 (STAT3) promotes neuron regeneration and functional recovery following SCI (5). In addition, connexin 43 (Cx43) expression has been demonstrated to aggravate secondary injury following SCI, leading to researchers proposing connexins as potential targets for SCI treatment (6).

Tropomyosin-related kinase B.T1 (trkB.T1), which is highly expressed in the nervous system of adult mammals, has been identified to accumulate in astrocytes, white matter and ependymal cells following SCI $(7,8)$. Although trkB.T1 lacks a kinase activation domain, it is active in signal transduction $(9,10)$. Wu et al $(11)$ performed whole genome analysis for trkB.T1 knockout (KO) mice and reported that trkB.T1 serves a critical role in SCI pain and progression by regulating pathways associated with the cell cycle (11).

Herein, a bioinformatic approach was used to analyze the microarray data compiled by Wu et al (11), in order to further investigate the differentially expressed genes (DEGs) in trkB.T1 KO mice at different time points following SCI. As genes with differential expression may be closely associated with SCI pathogenesis, the functions and interactions of the identified DEGs were explored. In addition, the present 
study aimed to identify potential target genes and their involvement in the biological functions underlying SCI pathogenesis.

\section{Materials and methods}

Data collection. Spinal cord tissues of SCI mice were profiled based on the Affymetrix Mouse Genome 4302.0 Array platform (11). Microarray data were provided by Wu et al (11); these data were generated from trkB.T1 KO and trkB.T1 wild type (WT) mice under different conditions, including sham operations, and at days 1, 3, and 7 following SCI. This dataset (GSE47681) was downloaded from the Gene Expression Omnibus database (www.ncbi.nlm.nih.gov/geo/).

Data preprocessing and DEG analysis. Differences in gene expression between trkB.T1 KO and trkB.T1 WT mice in sham groups (sham KO vs. sham WT) and in SCI groups at days 1,3 , and 7 after injury (day $1 \mathrm{KO}$ vs. day $1 \mathrm{WT}$; day 3 $\mathrm{KO}$ vs. day $3 \mathrm{WT}$; and day $7 \mathrm{KO}$ vs. day $7 \mathrm{WT}$ ) were respectively compared via unpaired t-tests using the $\mathrm{R}$ package 'limma' (12). Genes for which met the $\mathrm{P}<0.05$ and $\log _{2}$ fold change (FC) $\geq 0.4$ cutoff points were selected as DEGs, following which gene expression profiles of DEGs were visualized via the 'gplots' in R package version 3.0. 1 (13).

Venn diagram analysis. In order to mine the feature genes from different datasets, a Venn diagram analysis was conducted using VennPlex version 1.0.0.2 software (www.irp.nia.nih. gov/bioinformatics/vennplex.html) (14). The DEGs and their respective $\log _{2} \mathrm{FC}$ values were uploaded to the VennPlex version 1.0.0.2 tool, from which differences in the expression levels of DEGs at several time points were obtained, and the number of upregulated, downregulated and contraregulated genes were calculated.

Co-expression module and functional analysis. Weighted correlation network analysis (WGCNA) was used to identify highly correlated genes based on gene expression patterns across the microarray samples (15). DEGs in trkB.T1 KO mice of the sham group and SCI groups at days 1, 3 and 7 following injury were subjected to co-expression analysis using the $\mathrm{R}$ package 'WGCNA' version 1.19 (15) based on the WGCNA algorithm. Significant gene co-expression modules were screened out using the clustering method. The Icorrelation coefficient $1 \geq 0.65$ and $\mathrm{P}<0.05$ were set as the cutoff values.

Gene Ontology (GO) (16) function and Kyoto Encyclopedia of Genes and Genomes (KEGG) (17) pathway analyses were conducted for the genes in the co-expression modules by the Database for Annotation, Visualization and Integrated Discovery (DAVID) online tool (18) with new fuzzy classification algorithms. GO and KEGG terms with counts $\geq 2$ and $\mathrm{P}<0.05$ was considered to indicate a statistically significant difference.

Protein-protein interaction network. PPI network analysis was performed to analyze the functional interactions between proteins encoded by DEGs associated with the co-expression network. the Search Tool for the Retrieval of Interacting Genes/Proteins (STRING) database (19) includes an extensive list of protein interacting pairs collected from neighborhood, gene fusion, co-occurrence, co-expression experiments, databases and text mining. Genes from the co-expression network were input into the STRING online tool to identify highly associated gene pairs. The protein pairs with medium confidence $(\geq 0.4)$ were collected and the PPI network was visualized using the package 'Cytoscape' version 3 (20). Significant nodes with high degrees of connectivity were screened out.

Module analysis. PPI networks contain several densely connected network modules, with genes in each module commonly involved in the same biological processes. ClusterONE (21) is a graph-clustering algorithm that incorporates weighted graphs and readily generates overlapping clusters (www.paccanarolab.org/cluster-one/). As such, ClusterONE version 1.0 was used to cluster the PPI network, with $\mathrm{P}<1 \times 10^{-4}$ set as the threshold value. Further KEGG pathway analysis of the module genes was subsequently undertaken to identify the significant pathways $(\mathrm{P}<0.05)$.

\section{Results}

Differential expression analysis. Based on $\mathrm{P}<0.05$ and $\log _{2} \mathrm{FCl}$ $\geq 0.4$, DEGs of trkB.T1 KO mice in the sham, day 1, day 3 and day $7 \mathrm{SCI}$ groups were respectively identified. As presented in Table I, the smallest number of DEGs occurred in the sham group, consisting of 45 upregulated and 21 downregulated genes. The day $3 \mathrm{SCI}$ group contained the largest number of differentially expressed genes, consisting of 206 upregulated and 149 downregulated genes. Gene expression profiles of the various DEGs in the different groups are presented in Fig. 1. This analysis suggested that DEG expression levels may be used to distinguish between trkB.T1 KO and WT samples.

Venn diagram analysis. The relationships between the different DEG groups are depicted in Fig. 2. Compared with the sham group, the number of overlapping DEGs of trkB.T1 KO mice at days 1, 3 and 7 after SCI were 7, 5 and 10, respectively. In addition, 26 DEGs overlapped between the day 1 and day 3 SCI groups; 17 overlapped between the day 1 and day 7 SCI groups; and 11 overlapped between the day 3 and day 7 SCI groups.

WGCNA co-expression analysis. A total of 664 DEGs in the sham group and SCI groups at days 1, 3, and 7 following injury were identified in trkB.T1 KO mice. Subjecting the DEGs to WGCNA analysis revealed that they were clustered into 11 modules (represented by the different colors in Fig. 3). The top four modules-those with the highest correlation coefficients $(\mathrm{CC})$ and lowest $\mathrm{P}$-values-were, in the following order: Magenta $\left(C C=0.66, P=3.67 \times 10^{-3}\right.$; gene count, 23), Purple $\left(\mathrm{CC}=0.65, \mathrm{P}=4.69 \times 10^{-3}\right.$; gene count, 15$)$, Brown $(\mathrm{CC}=0.9$, $\mathrm{P}=7.20 \times 10-7$; gene count, 96$)$ and Blue $\left(\mathrm{CC}=0.88, \mathrm{P}=3.62 \times 10^{-6}\right.$; gene count, 110) modules.

Functional analysis revealed that the genes in the Magenta module were significantly enriched in 'response to virus', 'immune response', and 'cytosolic DNA-sensing pathway'; genes in the Purple module were strongly associated with 'extracellular region' and 'drug metabolism'; genes in the Blue module were significantly associated with 'immune response', 'Fc $\gamma$ R-mediated phagocytosis' and 'complement and 
Table I. Differentially expressed genes in the sham and spinal cord injury groups.

\begin{tabular}{lccrr}
\hline Group & $\begin{array}{c}\text { Upregulated } \\
\text { gene count }\end{array}$ & $\begin{array}{c}\text { Mean value } \\
\text { logFC (up) }\end{array}$ & $\begin{array}{c}\text { Downregulated } \\
\text { gene count }\end{array}$ & $\begin{array}{c}\text { Mean value } \\
\operatorname{logFC}(\text { down) }\end{array}$ \\
\hline Sham KO & 45 & 0.882 & 21 & -0.5569 \\
Day 1 KO & 73 & 0.7096 & 132 & -0.6407 \\
Day 3 KO & 206 & 0.6201 & 149 & -0.8843 \\
Day 7 KO & 74 & 0.54 & 32 & -0.5852 \\
\hline
\end{tabular}

KO, knockout; FC, fold change.

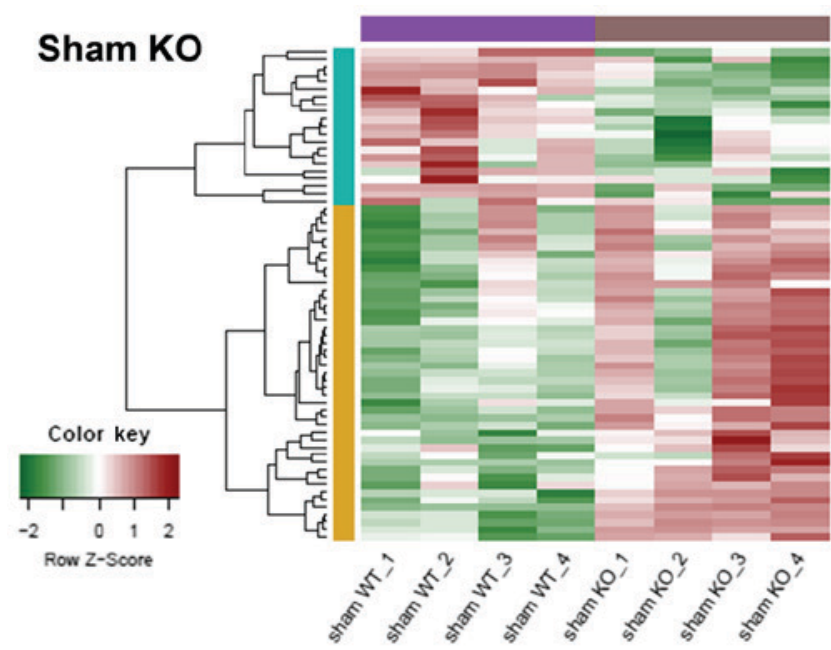

Day 3 KO

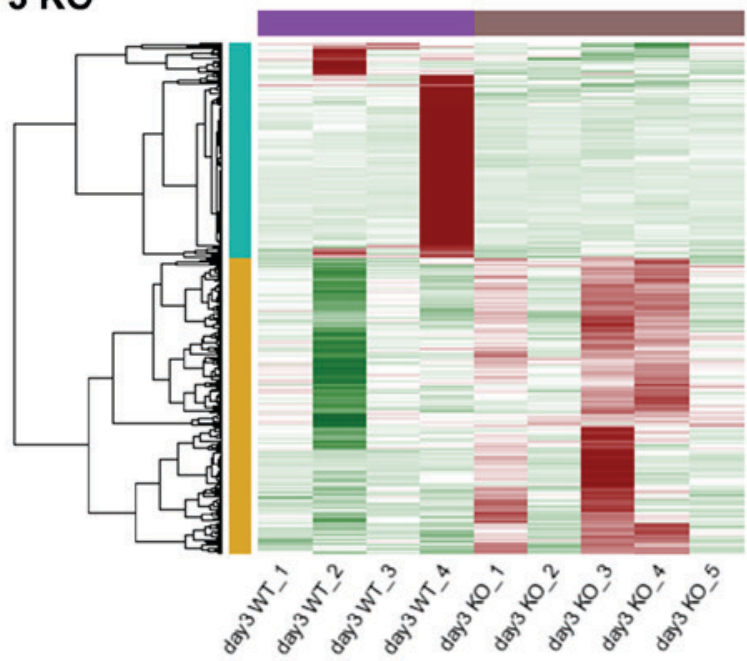

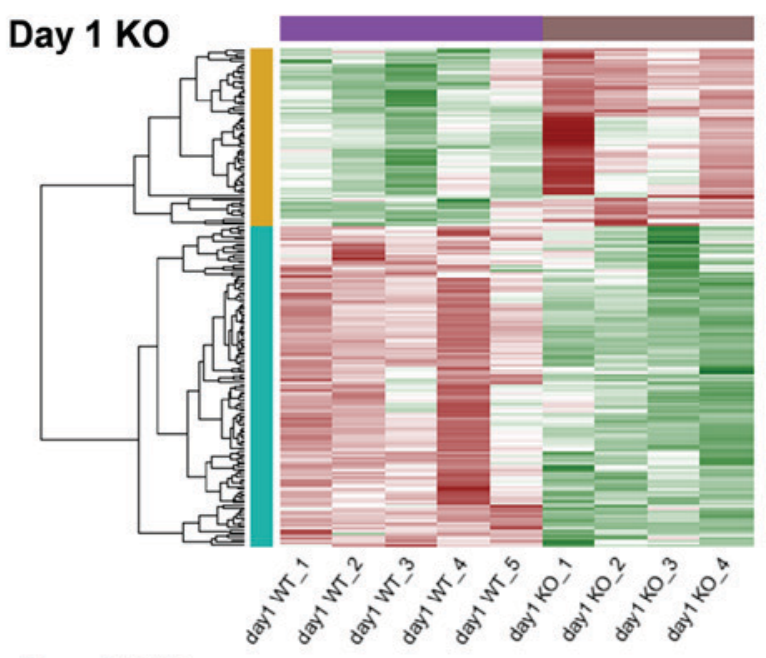

Day 7 KO

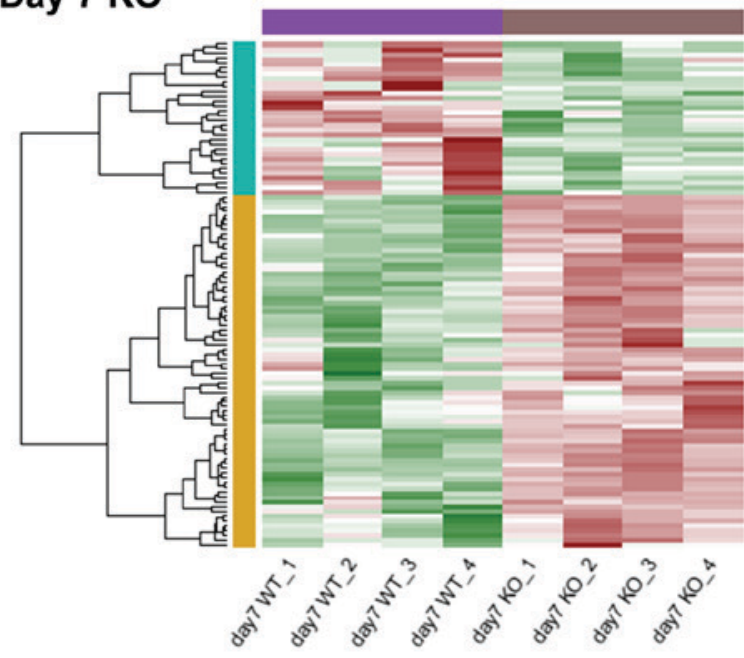

Figure 1. Heat map of the differentially expressed genes in trkB.T1 KO mice following injury at different time points. Red represents relatively high levels of expression; green represents relatively low levels of expression. Purple and brown bars indicated the trkB.T1 WT samples and trkB.T1 KO samples, respectively; yellow and blue bars indicated that upregulated and downregulated genes respectively in trkB.T1 KO mice following injury. The heat map demonstrated that the samples from trkB.T1 KO were distinct from those from the trkB.T1 WT mice. KO, knockout; WT, wild type.

coagulation cascades'; and genes in the Brown module were markedly enriched in 'oxidation reduction', 'drug metabolism' and 'complement and coagulation cascades' (Table II).

PPI construction and module analysis. The PPI network connected 161 nodes through 1,051 edges; the color scheme used for the nodes was the same as that used for the modules described in the previous section (Fig. 4). The top 20 nodes (Table III) were determined to have a degree $\geq 28$ in the PPI network, including protein tyrosine phosphatase, receptor type C (PTPRC; degree, 43; Blue), coagulation factor II, thrombin $(F 2$; degree, 41 ; Brown), plasminogen ( $P L G$; degree, 38; Blue), and thymocyte selection associated family member 2 (Themis2; degree, 37; Blue).

ClusterOne analysis resulted in three significant gene clusters (Fig. 5), and the DAVID online tool, which facilitates 
Table II. Significant GO and KEGG pathways for genes in co-expression modules.

\begin{tabular}{|c|c|c|c|c|}
\hline Module & Category & Term & Count & P-value \\
\hline \multirow[t]{9}{*}{ Magenta } & \multirow[t]{2}{*}{$\mathrm{BP}$} & GO:0009615 response to virus & 5 & $1.85 \times 10^{-7}$ \\
\hline & & GO:0006955 immune response & 6 & $1.07 \times 10^{-5}$ \\
\hline & $\mathrm{BP}$ & GO:0006952 defense response & 5 & $2.09 \times 10^{-4}$ \\
\hline & $\mathrm{CC}$ & GO:0005783 endoplasmic reticulum & 5 & $5.95 \times 10^{-4}$ \\
\hline & \multirow[t]{2}{*}{$\mathrm{MF}$} & GO:0032555 purine ribonucleotide binding & 8 & $1.17 \times 10^{-3}$ \\
\hline & & GO:0032553 ribonucleotide binding & 8 & $1.17 \times 10^{-3}$ \\
\hline & MF & GO:0017076 purine nucleotide binding & 8 & $1.50 \times 10^{-3}$ \\
\hline & \multirow[t]{2}{*}{ KEGG_PATHWAY } & mmu04623:Cytosolic DNA-sensing pathway & 3 & $1.84 \times 10^{-3}$ \\
\hline & & mmu04622:RIG-I-like receptor signaling pathway & 3 & $2.80 \times 10^{-3}$ \\
\hline \multirow[t]{3}{*}{ Purple } & \multirow[t]{2}{*}{$\mathrm{CC}$} & GO:0044421 extracellular region part & 4 & $1.50 \times 10^{-2}$ \\
\hline & & GO:0005576 extracellular region & 5 & $2.34 \times 10^{-2}$ \\
\hline & KEGG_PATHWAY & mmu00983:Drug metabolism & 2 & $4.11 \times 10^{-2}$ \\
\hline \multirow[t]{12}{*}{ Blue } & \multirow[t]{2}{*}{$\mathrm{BP}$} & GO:0006955 immune response & 15 & $8.03 \times 10^{-8}$ \\
\hline & & GO:0009611 response to wounding & 11 & $9.24 \times 10^{-6}$ \\
\hline & $\mathrm{BP}$ & GO:0001775 cell activation & 9 & $3.32 \times 10^{-5}$ \\
\hline & \multirow[t]{2}{*}{$\mathrm{CC}$} & GO:0034358 plasma lipoprotein particle & 4 & $4.65 \times 10^{-4}$ \\
\hline & & GO:0032994 protein-lipid complex & 4 & $4.65 \times 10^{-4}$ \\
\hline & $\mathrm{CC}$ & GO:0005886 plasma membrane & 28 & $3.60 \times 10^{-3}$ \\
\hline & \multirow[t]{2}{*}{ MF } & GO:0001871 pattern binding & 6 & $4.28 \times 10^{-4}$ \\
\hline & & GO:0030247 polysaccharide binding & 6 & $4.28 \times 10^{-4}$ \\
\hline & $\mathrm{MF}$ & GO:0005539 glycosaminoglycan binding & 5 & $2.46 \times 10^{-3}$ \\
\hline & \multirow[t]{3}{*}{ KEGG_PATHWAY } & mmu04666:Fc $\gamma$ R-mediated phagocytosis & 6 & $3.71 \times 10^{-4}$ \\
\hline & & mmu04610:Complement and coagulation cascades & 5 & $1.28 \times 10^{-3}$ \\
\hline & & mmu04650:Natural killer cell mediated cytotoxicity & 5 & $7.47 \times 10^{-3}$ \\
\hline \multirow[t]{12}{*}{ Brown } & \multirow[t]{2}{*}{$\mathrm{BP}$} & GO:0055114 oxidation reduction & 10 & $2.03 \times 10^{-3}$ \\
\hline & & GO:0002526 acute inflammatory response & 4 & $4.97 \times 10^{-3}$ \\
\hline & BP & GO:0055090 acylglycerol homeostasis & 2 & $8.52 \times 10^{-3}$ \\
\hline & \multirow[t]{3}{*}{$\mathrm{CC}$} & GO:0005576 extracellular region & 22 & $7.15 \times 10^{-5}$ \\
\hline & & GO:0005615 extracellular space & 10 & $1.23 \times 10^{-3}$ \\
\hline & & GO:0044421 extracellular region part & 12 & $1.97 \times 10^{-3}$ \\
\hline & \multirow[t]{2}{*}{ MF } & $\begin{array}{l}\text { GO:0016712 oxidoreductase activity, acting on paired } \\
\text { donors, with incorporation or reduction of molecular } \\
\text { oxygen, reduced flavin or flavoprotein as one donor, } \\
\text { and incorporation of one atom of oxygen }\end{array}$ & 4 & $9.68 \times 10^{-4}$ \\
\hline & & GO:0009055 electron carrier activity & 6 & $1.99 \times 10^{-3}$ \\
\hline & MF & GO:0043176 amine binding & 4 & $2.49 \times 10^{-3}$ \\
\hline & \multirow[t]{3}{*}{ KEGG_PATHWAY } & mmu00982:Drug metabolism & 5 & $1.04 \times 10^{-3}$ \\
\hline & & mmu04610:Complement and coagulation cascades & 4 & $1.04 \times 10^{-2}$ \\
\hline & & mmu00591:Linoleic acid metabolism & 3 & $3.16 \times 10^{-2}$ \\
\hline
\end{tabular}

MF, molecular function; BP, biological process; CC, cellular component; KEGG, Kyoto Encyclopedia of Genes and Genomes.

classification of functionally associated genes in different KEGG pathways (Table IV), demonstrated that the genes in cluster 1 were closely associated with 'complement and coagulation cascades', 'drug metabolism', and 'phenylalanine, tyrosine, and tryptophan biosynthesis'; the genes in cluster 2 were significantly involved in 'Fc $\gamma$ R-mediated phagocytosis', 'B cell receptor signaling pathway' and 'natural killer cell mediated cytotoxicity'; genes in cluster 3 were involved in the 'cytosolic DNA-sensing' and 'RIG-I-like receptor signaling pathways'.

\section{Discussion}

SCI often results in chronic pain and loss of physical function (22). A previous study demonstrated that TrkB.T1, as a 
Table III. Top 20 genes with the highest degree in the PPI network.

\begin{tabular}{lll}
\hline Gene & Module type & Degree \\
\hline PTPRC & Blue & 43 \\
$F 2$ & Brown & 41 \\
PLG & Blue & 38 \\
THEMIS & Blue & 37 \\
SERPINC1 & Blue & 35 \\
SLC27A5 & Blue & 34 \\
SLC15A3 & Blue & 33 \\
AKR1C6 & Brown & 32 \\
CD53 & Blue & 32 \\
TRIM30A & Magenta & 32 \\
PTPN6 & Blue & 32 \\
GPR65 & Blue & 32 \\
SLCO1B2 & Blue & 32 \\
APOF & Brown & 32 \\
FCGR4 & Blue & 30 \\
AIF1 & Brown & 30 \\
APOA5 & Brown & 28 \\
IFI47 & Magenta & 28 \\
FGG & Brown & 28 \\
FCGR1 & Blue & 28 \\
\end{tabular}

receptor for brain-derived neurotrophic factor, serves a critical role in neuropathic pain and SCI progression (11). In the present study, the significant DEGs at different time points following SCI were identified and potential targets for SCI therapy were suggested based on the differential expression profiles induced by TrkB.T1 KO.

In total, 664 genes were differentially expressed in the sham group and SCI groups at different time points following injury. Gene expression profiles of the DEGs differed significantly between TrkB.T1 KO and TrkB.T1 WT samples, and construction of a Venn diagram indicated a lower number of overlapping DEGs under the different conditions. Based on these results, it was concluded that the DEGs screened out were significant.

Analysis of the PPI network suggested that PTPRC, F2, and $P L G$ were the most significant nodes, with multiple interactions with other nodes, and PTPRC with a degree of 43 was identified to be the single most significant node in the PPI network. Previous research has shown PTPRC to be a critical DEG in the PPI network following spared nerve injury, and PTPRC might thus represent a potential target for peripheral neuropathic pain intervention (23). Based on the results obtained in the present study, it was speculated that PTPRC may also have a critical role in the pathology of SCI.

Protein tyrosine phosphatase, the receptor type $\mathrm{C}$ encoded by $P T P R C$, is a member of the protein tyrosine phosphatase family. PTPRC, also known as CD45 antigen, is highly expressed in hematopoietic cells in particular and is strongly associated with cellular growth and proliferation. Transplanted hematopoietic stem cells have been demonstrated to persist in SCI lesions and contribute to functional recovery following

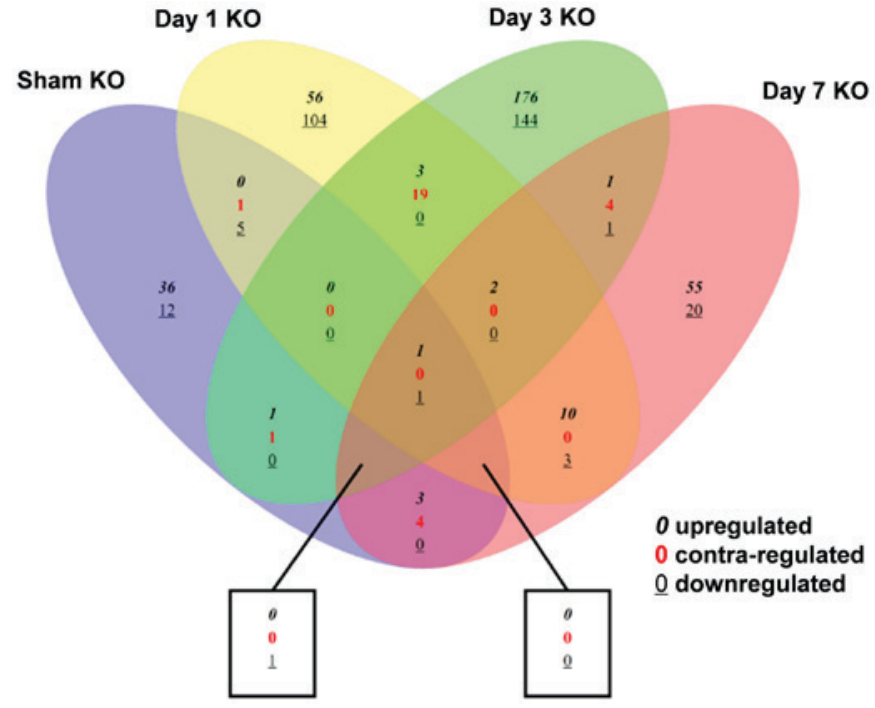

Figure 2. Venn diagram of the differentially expressed genes among the different groups. KO, knockout.

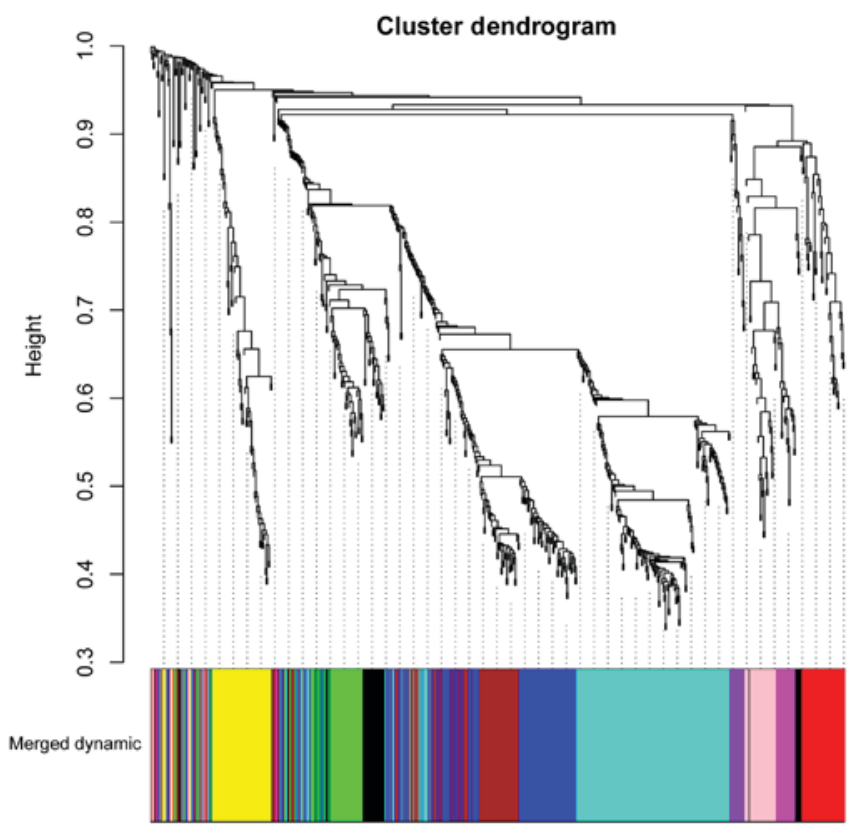

Figure 3. Hierarchical clustering tree for genes with co-expression interactions. The branches of the dendrogram indicate the gene clusters; co-expressed modules are represented by different colors.

SCI (24). The expression of CD45 has been revealed to be upregulated in macrophages and microglia during the inflammatory response following SCI (25). The anti-inflammatory activation of macrophages and microglia promotes tissue and function repair following SCI (25).

In addition, PTPRC in cluster 2 was significantly enriched in Fc $\gamma$ R-mediated phagocytosis, a finding consistent with previous results; Jin et al (26) also reported that the genes upregulated post-SCI are significantly enriched in Fc $\gamma$ R-mediated phagocytosis. Furthermore, Ohri et al (27) observed that the Fc $\gamma$ R-mediated phagocytosis pathway is dysregulated in C/EBP-homologous protein 10-null mice following severe SCI. Previous evidence further suggests that superoxide is produced during Fc $\gamma$ R-mediated 
Table IV. Significant pathways for genes in the protein-protein interaction network.

\begin{tabular}{|c|c|c|c|}
\hline Cluster & Term & P-value & Genes \\
\hline \multirow[t]{3}{*}{ Cluster 1} & mmu04610: Complement and coagulation cascades & $1.02 \times 10^{-8}$ & $\begin{array}{l}F 13 B, M B L 2, F G G, H C, \text { SERPINF } 2, \\
F 2, \text { SERPINC1, PLG }\end{array}$ \\
\hline & mmu00982: Drug metabolism & $3.15 \times 10^{-3}$ & $\begin{array}{l}C Y P 2 D 9, C Y P 2 A 12, C Y P 2 D 10 \\
C Y P 2 D 26\end{array}$ \\
\hline & mmu00400: Phenylalanine, tyrosine and tryptophan biosynthesis & $1.99 \times 10^{-2}$ & PAH, TAT \\
\hline \multirow[t]{5}{*}{ Cluster 2} & mmu04666: Fc $\gamma$ R-mediated phagocytosis & $1.24 \times 10^{-4}$ & PTPRC, NCF1, PLCG2, WAS, FCGR1 \\
\hline & mmu04662: B cell receptor signaling pathway & $1.28 \times 10^{-3}$ & PTPN6, PLCG2, CD72, BLNK \\
\hline & mmu04650: Natural killer cell mediated cytotoxicity & $4.29 \times 10^{-3}$ & CD48, PTPN6, PLCG2, FCGR4 \\
\hline & mmu05340: Primary immunodeficiency & $4.35 \times 10^{-3}$ & $P T P R C, I L 7 R, B L N K$ \\
\hline & mmu04670: Leukocyte transendothelial migration & $4.23 \times 10^{-2}$ & $C Y B B, N C F 1, P L C G 2$ \\
\hline \multirow[t]{2}{*}{ Cluster 3} & mmu04623: Cytosolic DNA-sensing pathway & $8.22 \times 10^{-6}$ & $D D X 58, T M E M 173, I R F 7, Z B P 1$ \\
\hline & mmu04622: RIG-I-like receptor signaling pathway & $1.57 \times 10^{-5}$ & DDX58, IFIH1, TMEM173, IRF7 \\
\hline
\end{tabular}

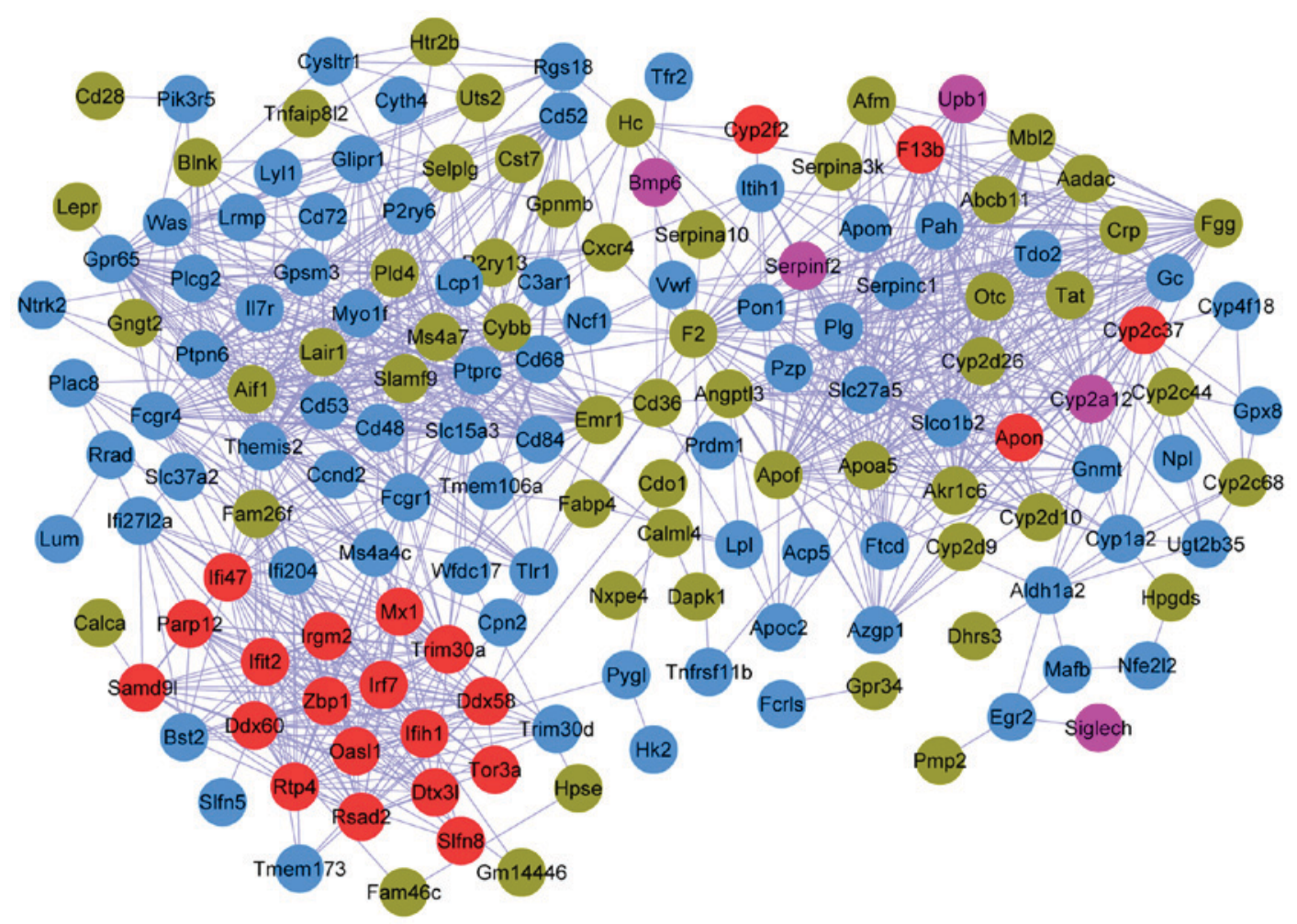

Figure 4. Protein-protein interaction network of the differentially expressed genes. Gene modules are represented by different colors.

phagocytosis, and is strongly associated with neuronal death following SCI $(28,29)$. In the present study, co-expression analysis also revealed that PTPRC was clustered in the Blue module, and the genes contained therein are known to be associated with Fc $\gamma$ R-mediated phagocytosis. Taken together, these results suggested that PTPRC serves a critical role in SCI progression via interactions with other genes.

$F 2$ and $P L G$ were also identified to be critical genes in the PPI network. PPI module analysis suggested that F2 and PLG composed cluster 2 , and were both significantly enriched in the complement and coagulation cascades. Co-expression analysis clustered F2 in the Brown module and PLG in the Blue module, and subsequent KEGG pathway analysis revealed that the genes in the Brown and Blue modules were closely associated with the complement and coagulation cascades, which suggested that the findings of the present study were of particular significance.

Coagulation factor II, also known as thrombin, is encoded by $F 2$. Thrombin functions in coagulation-associated reactions and thus in reducing blood loss. Like thrombin, plasmin encoded by $P L G$ is a serine protease present in the blood (27). It has been reported that patients with SCI are often afflicted with coagulation-related disorders, including altered platelet function and coagulation factor concentrations, which may lead to cardiovascular disorders $(30,31)$. Therefore, focused targeting of $F 2$ and $P L G$ expression may aid in the mitigation of cardiovascular disorders associated with SCI. 


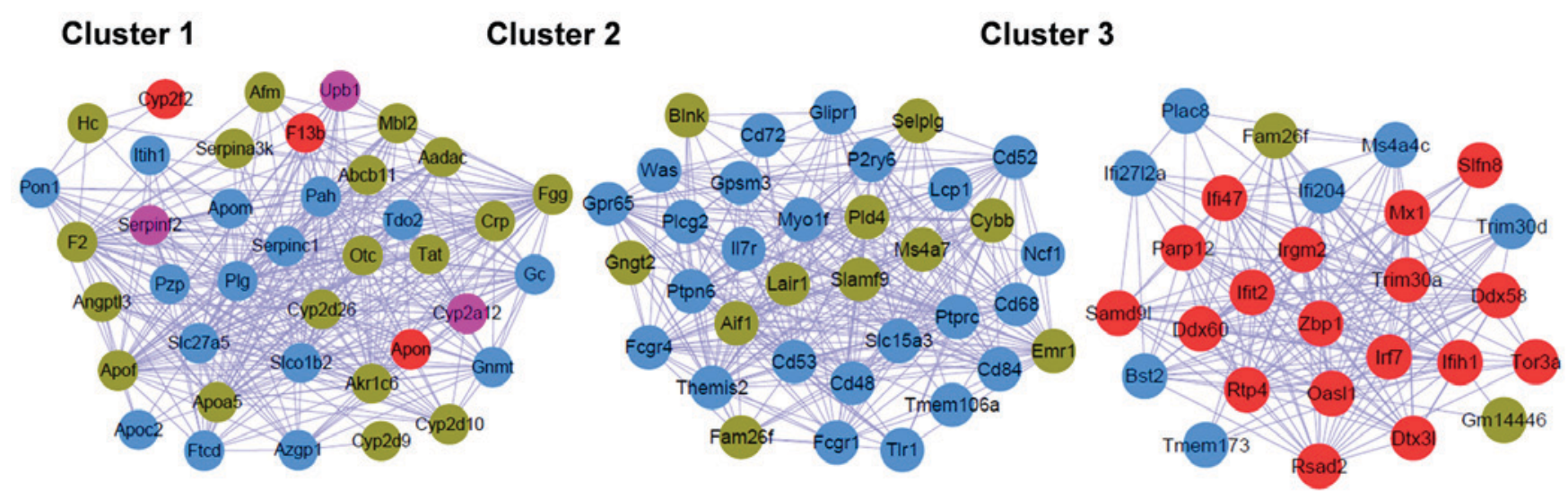

Figure 5. Significant modules of the protein-protein interaction network. Gene modules are represented by different colors.

Due to a lack of clinical materials and funding, the present study was unable to provide experimental validation of these findings, but these results warrant future research involving both cellular and clinical samples.

In conclusion, PTPRC, F2 and $P L G$ were identified as significant nodes in the PPI network, and therefore may have critical function in SCI progression through their involvement in inflammatory responses and coagulation disorders. As such, PTPRC, F2 and PLG may be candidate targets for SCI gene therapy. The findings of the present study may lead to a better understanding of the pathogenesis of SCI and shed light on the identification of novel therapeutic targets. However, clinical trials on gene therapy are necessary to assess potential genetic strategies for SCI.

\section{Acknowledgments}

Not applicable.

\section{Funding}

The study was funded by the Shanghai Science and Technology Committee program (no 16411955200) and the Scientific Research and Innovation Team Funding Plan of Shanghai Sanda University.

\section{Availability of data and materials}

All data generated or analyzed during the present study are included in this published article.

\section{Authors' contributions}

LW and FH were responsible for the conception and design of the research, and drafting the manuscript. WZ performed the data acquisition. WC performed the data analysis and interpretation. BY participated in the design of the study and performed the statistical analysis. All authors have read and approved the manuscript.

\section{Ethics approval and consent to participate}

Not applicable.

\section{Patient consent for publication}

Not applicable.

\section{Competing interests}

The authors declare that they have no competing interests.

\section{References}

1. Crewe NM and Krause JS: Spinal cord injury. In: Medical, Psychosocial and Vocational Aspects of Disability. Brodwin MG, Siu FW, Howard J and Brodwin ER (eds). 3rd edition. Elliott and Fitzpatrick, Inc., Athens, GA, pp 289-304, 2009.

2. Wyndaele $M$ and Wyndaele JJ: Incidence, prevalence and epidemiology of spinal cord injury: What learns a worldwide literature survey? Spinal Cord 44: 523-529, 2006.

3. Chen Y, Tang Y, Vogel LC and DeVivo MJ: Causes of spinal cord injury. Top Spinal Cord Inj Rehabil 19: 1-8, 2013.

4. Shah RR and Tisherman SA: Spinal cord injury. J Imaging the ICU Patient: 377-380, 2014.

5. Lang C, Bradley PM, Jacobi A, Kerschensteiner M and Bareyre FM: STAT3 promotes corticospinal remodelling and functional recovery after spinal cord injury. EMBO Rep 14: 931-937, 2013.

6. Huang C, Han X, Li X, Lam E, Peng W, Lou N, Torres A, Yang M, Garre JM, Tian GF, et al: Critical role of connexin 43 in secondary expansion of traumatic spinal cord injury. J Neurosci 32: 3333-3338, 2012.

7. King VR, Bradbury EJ, McMahon SB and Priestley JV: Changes in truncated trkB and $\mathrm{p} 75$ receptor expression in the rat spinal cord following spinal cord hemisection and spinal cord hemisection plus neurotrophin treatment. Exp Neurol 165: 327-341, 2000.

8. Liebl DJ, Huang W, Young W and Parada LF: Regulation of Trk receptors following contusion of the rat spinal cord. Exp Neurol 167: 15-26, 2001.

9. Dorsey SG, Renn CL, Carimtodd L, Barrick CA, Bambrick L, Krueger BK, Ward CW and Tessarollo L: In vivo restoration of physiological levels of truncated TrkB.T1 receptor rescues neuronal cell death in a trisomic mouse model. Neuron 51: 21-28, 2006.

10. Carim-Todd L, Bath KG, Fulgenzi G, Yanpallewar S, Jing D, Barrick CA, Becker J, Buckley H, Dorsey SG, Lee FS and Tessarollo L: Endogenous truncated TrkB.T1 receptor regulates neuronal complexity and TrkB kinase receptor function in vivo. J Neurosci 29: 678-685, 2009.

11. Wu J, Renn CL, Faden AI and Dorsey SG: TrkB. T1 contributes to neuropathic pain after spinal cord injury through regulation of cell cycle pathways. J Neurosci 33: 12447-12463, 2013.

12. Smyth GK: Limma: Linear models for microarray data. J Bioinform Comput Biol Solut Using R and Bioconductor: 397-420, 2005. 
13. Warnes GR, Bolker B, Bonebakker L, Gentleman R, Liaw WHA Lumley T, Maechler M, Magnusson A, Moeller S, Schwartz M and Venables B: gplots: Various R programming tools for plotting data. $\mathrm{R}$ package version 3.0. 1. The Comprehensive $\mathrm{R}$ Archive Network, 2016.

14. Cai H, Chen H, Yi T, Daimon CM, Boyle JP, Peers C, Maudsley S and Martin B: VennPlex-A novel Venn diagram program for comparing and visualizing datasets with differentially regulated datapoints. PLoS One 8: e53388, 2013.

15. Langfelder P and Horvath S: WGCNA: An R package for weighted correlation network analysis. BMC Bioinformatics 9: 559, 2008.

16. Ashburner M, Ball CA, Blake JA, Botstein D, Butler H, Cherry JM, Davis AP, Dolinski K, Dwight SS, Eppig JT, et al: Gene ontology: Tool for the unification of biology. The gene ontology consortium. Nat Genet 25: 25-29, 2000.

17. Kanehisa M and Goto S: KEGG: Kyoto encyclopedia of genes and genomes. Nucleic Acids Res 28: 27-30, 2000.

18. Huang da W, Sherman BT and Lempicki RA: Systematic and integrative analysis of large gene lists using DAVID bioinformatics resources. Nat Protoc 4: 44-57, 2009.

19. Szklarczyk D, Franceschini A, Wyder S, Forslund K, Heller D, Huerta-Cepas J, Simonovic M, Roth A, Santos A, Tsafou KP, et al: STRING v10: Protein-protein interaction networks, integrated over the tree of life. Nucleic Acids Res 43: D447-D452, 2015.

20. Shannon P, Markiel A, Ozier O, Baliga NS, Wang JT, Ramage D, Amin N, Schwikowski B and Ideker T: Cytoscape: A software environment for integrated models of biomolecular interaction networks. Genome Res 13: 2498-2504, 2003.

21. Nepusz T, Yu H and Paccanaro A: Detecting overlapping protein complexes in protein-protein interaction networks. Nat Methods 9: 471-472, 2012.

22. Defrin R, Ohry A, Blumen N and Urca G: Characterization of chronic pain and somatosensory function in spinal cord injury subjects. Pain 89: 253-263, 2001.

23. Yang YK, Lu XB, Wang YH, Yang MM and Jiang DM: Identification crucial genes in peripheral neuropathic pain induced by spared nerve injury. Eur Rev Med Pharmacol Sci 18: 2152-2159, 2014.
24. Koda M, Okada S, Nakayama T, Koshizuka S, Kamada T, Nishio Y, Someya Y, Yoshinaga K, Okawa A, Moriya H and Yamazaki M: Hematopoietic stem cell and marrow stromal cell for spinal cord injury in mice. Neuroreport 16: 1763-1767, 2005.

25. David S and Kroner A: Repertoire of microglial and macrophage responses after spinal cord injury. Nat Rev Neurosci 12: 388-399, 2011.

26. Jin $\mathrm{L}, \mathrm{Wu} \mathrm{Z}, \mathrm{Xu} \mathrm{W}, \mathrm{Hu} X$, Zhang J, Xue Z and Cheng L: Identifying gene expression profile of spinal cord injury in rat by bioinformatics strategy. Mol Biol Rep 41: 3169-3177, 2014

27. Ohri SS, Maddie MA, Zhang Y, Shields CB, Hetman M and Whittemore SR: Deletion of the pro-apoptotic endoplasmic reticulum stress response effector CHOP does not result in improved locomotor function after severe contusive spinal cord injury. J Neurotrauma 29: 579-588, 2012.

28. Xu W, Chi L, Xu R, Ke Y, Luo C, Cai J, Qiu M, Gozal D and Liu R: Increased production of reactive oxygen species contributes to motor neuron death in a compression mouse model of spinal cord injury. Spinal Cord 43: 204-213, 2005.

29. Ueyama T, Lennartz MR, Noda Y, Kobayashi T, Shirai Y, Rikitake K, Yamasaki T, Hayashi S, Sakai N, Seguchi H, et al: Superoxide production at phagosomal cup/phagosome through $\beta \mathrm{I}$ protein kinase $\mathrm{C}$ during $\mathrm{Fc} \gamma \mathrm{R}$-mediated phagocytosis in microglia. J Immunol 173: 4582-4589, 2004.

30. Furlan JC and Fehlings MG: Cardiovascular complications after acute spinal cord injury: Pathophysiology, diagnosis, and management. Neurosurg Focus 25: E13, 2008.

31. Bravo G, Guízar-Sahagún G, Ibarra A, Centurión D and Villalón CM: Cardiovascular alterations after spinal cord injury: An overview. Curr Med Chem Cardiovasc Hematol Agents 2: 133-148, 2004.

This work is licensed under a Creative Commons Attribution-NonCommercial-NoDerivatives 4.0 International (CC BY-NC-ND 4.0) License. 